\title{
Theory of Mind Deficit and Its Neural Dysfunction in Schizophrenia
}

\author{
Rui Liang ${ }^{1, *, \dagger}$ Qingjie Meng ${ }^{2, *, \dagger}$ Jingying $\mathrm{Nie}^{3, *, \dagger}$
}

\author{
${ }^{1}$ Sanxin Bilingual School, Zhongshan, Guangdong, 528463, China \\ ${ }^{2}$ Ridley College, St. Catharines, Ontario, L2R7C3, Canada \\ ${ }^{3}$ College Art and Science, New York University, New York, NY, 10003, US \\ †These authors contributed equally. \\ *Corresponding author.Email: ${ }^{1} 767986427 @ q q . c o m,{ }^{2} 1042809956 @ q q . c o m,{ }^{3} j n 2261 @ n y u . e d u$
}

\begin{abstract}
Social cognition refers to how people perceive themselves and others in the social world (including human perception, causal attribution of self and others, and the process of bringing social judgment into decision-making). Theory of Mind (ToM) refers to an individual' s ability to understand others' mental state. These two abilities bring people a better understanding of others and life. Without these two important components, people may soon fall into the predicament of social interaction. However, schizophrenia is a destructive mental illness that damages mental and social functions and often leads to the development of comorbidities. Nowadays, as researchers mainly tap into the symptoms of schizophrenia, theory of mind doesn't get enough attention, especially to social cognition. Shown in our paper will be the development of social skills and the relationship between theory of mind and schizophrenia. This article shows The development of social skills and the relationship between theory of mind and schizophrenia. This essay will delve into three aspects: 1) whether the deficit exists in the theory of mind; 2) Different levels in mentalizing in individuals with schizophrenia; 3) and the effect of damage of neuron to ToM in schizophrenia. There have been many studies on schizophrenia, but there are still some limitations. From a previous review of existing studies, we found that patients with schizophrenia have a deficit in ToM processing, and this deficit is related to dysfunction of certain brain areas. However, the relationship between the deficit in ToM processing and brain dysfunction is not clear yet. Future studies can be focused on the possibilities of treatment restoring dysfunctional brain regions or exercise improving ToM processing. Chronical studies are necessary to study the natural changes in neural activity and ToM development in patients and the human intervention's consequence.
\end{abstract}

Keywords: Review study, Schizophrenia, Theory of mind, Social cognition, Neural dysfunction in schizophrenia

\section{INTRODUCTION}

Theory of mind (ToM) refers to an individual's ability to understand other's mental states. It is advantageous to predict others' behaviors, thoughts, and emotions in social interactions. The most featured test is the false-belief task, which examines participants' ability to think from others' perspectives. If children can imagine an other's perspective and correctly predict resulted behavior while they are on a third party's perspective, they are seen to complete the false-believe task; on the contrary, if they predict an other's behavior based on what they see, that is a failure on the task.
Experiments have shown, most children develop ToM between 3-5 years old and start succeeding falsebelieve tasks from 4 . Pre-schoolers start understanding the complexity of emotions, such as the inconsistency between expressed emotions and real emotions, changing emotions due to different situations, and the mixture of various emotions at the same time [1]. A significant change in social interaction that influenced by this change of ToM is that, from this period, children begin to prefer contact with other preschoolers instead of adult caregivers [2].

Social interactions are also an influential aspect of the development of the theory of mind. Researches have found that, during children's development, family size, 
language development, friendship all together cultivate their theory of mind. Theories include environments as a trigger to ToM development, "theory theory" (children develop their ToM to process information from new social experiences), "simulation theory" (pretend to play in the different social environment brings children different understanding which causes the individual difference in ToM development), and executive function hypothesis (behavioral functions influence development of ToM) [3-6]. Therefore disabilities (e.g., blindness, deafness) can be obstacles to the theory of mind development and social competence. In many researches, patients with schizophrenia cannot usually understand other perspectives [7]. Worse performance in a social context also represents their weaker theory of mind capacity. Social cognition (i.e., how people perceive themselves and other people in the social world) includes human perception, causal attribution of self and others, and bringing social judgment into decision-making. Social cognition research shares 4 common features. The first is unabashed mentalism, focusing on mental representations such as "schemas." The second common feature is that social cognition is process-oriented. Third, the characteristic of social cognition is the mutual transformation of interdisciplinary methods and concepts. Society has obvious integration with traditional cognitive psychology and other fields such as developmental psychology, clinical psychology, and neuroscience. Finally, social cognition researchers focus on practical applications.

Schizophrenia is a destructive mental illness that damages mental and social functions and often leads to comorbidities. According to the American Psychiatric Association's standard, schizophrenia is characterized by positive and negative symptoms that may affect the patient's thoughts, perception, speech, emotion, and behavior. Positive symptoms include hallucinations, the voice of talking to or with the patient, and delusions. Negative symptoms include depression, pleasure, loss of will or motivation, and social withdrawal. Schizophrenia is also characterized by confusion in thinking, manifested in speech and behavior. The accidental discovery of antipsychotics gave rise to the first scientifically credible pathophysiological theory of schizophrenia, the dopamine hypothesis [8]. The neurodevelopmental hypothesis of schizophrenia assumes that the etiology and etiology (such as genetic and environmental insults) precede the disease's onset and disrupt the normal brain development process, leading to subtle changes in specific cells, circuits, and their connectivity [9-11]. This interruption of development makes a person prone to malfunction and then shows symptoms of a disease diagnosis. However, in contrast to other inherited neurodevelopmental disorders, the phenotype of schizophrenia does not extend until the incubation period extends to the second and third decade of life. From the onset to the disease course, the disease persists in the form of static encephalopathy, and only brain abnormalities develop during the aging process [12].

First, compared with non-clinical controls, patients with schizophrenia show defects. Second, these deficiencies are more serious than those with other mental illnesses, such as depression. Third, the biggest flaw is reflected in the perception of negative emotions. Fourth, the deficit in emotional perception is stable over time, although there is evidence that people in remission may perform better than people in the disease's acute phase. Fifth, people with schizophrenia do not perform well in judging what a person is thinking or feeling but are less impaired in social judgments such as more specific determination of what a person is wearing or what a person is doing. Sixth, many patients with schizophrenia have limited visual scanning when performing emotional perception tasks and spend less time checking for obvious facial features. Finally, emotional perception disorders appear in the early stages of the disorder [13,14]. Attribution refers to explaining why people have positive and negative events in their lives [15-17]. Usually, people attribute positive events to themselves and the responsibility for negative events to others. Much of the work on schizophrenia has focused on the attribution style of paranoid or persecutory delusions. They tend to attribute negative events to others instead of blaming the situation on others. This attribution method is called "personalized bias." According to the research of Bentall et al., Two factors can prevent people suffering from persecution delusions from correcting their prejudices when faced with uncertain situational information: a strong need for closure (unclear tolerance) and ToM damage [18]. Preliminary research has shown the link between the need for closure and the delusion of persecution and the link between ToM deficiencies and personalized attribution [19]. Of course, people with persecution delusions may also have other social cognitive biases, such as the tendency to draw a direct conclusion and prove that there is a confirmation bias (i.e., seeking confirmatory evidence for a belief rather than disconfirmatory evidence) [20$22]$. In general, various social cognitive deficiencies and prejudices can lead to paranoia.

In short, cultivating a good ToM plays an important role in persons in life, whatever they are for society, friends, or families. Similarly, social cognition is the primary function that enables people to socialize and understand each other. Without two significant parts in persons, they may soon get into difficulties in social interaction. Nowadays, as researchers mainly tap into the symptoms of schizophrenia, theory of mind doesn't get enough attention, especially to social cognition. Our paper will be the development of social skills and the relationship between theory of mind and schizophrenia. 
This essay will delve into three aspects: 1) whether the deficit exists in the theory of mind; 2) Different levels in mentalising in individuals with schizophrenia; 3) and the effect of damage of neuron to ToM in schizophrenia.

\section{COGNITIVE AND AFFECTIVE TOM IN SCHIZOPHRENIA}

Theory of mind disorders are common in patients with schizophrenia and are related to an impaired social function. Social development research shows a deep connection between cognitive insight and cognitive TOM, but cognitive incite is very different from cognitive TOM. Cognitive insight involves the metacognitive process of correcting distortions and reassessment experiences and includes the dimensions of overconfidence in beliefs and self-reflection. Still, TOM is about understanding thoughts, desires, and intentions from others [23]. Hinting Task was used to evaluate ToM [24]. On the "Hinting Task", participants explained others' intentions through 10 short aural videos of the social interaction between the two characters. The results showed that schizophrenic patients were unable to make judgments about emotions or made wrong judgments about emotions due to the influence of TOM.

To see what caused the schizophrenic patients' emotional judgment errors, we conducted investigations on cognitive insight and TOM. To assess insight, experimenters used the Birchwood insight scale and the Beck cognitive insight scale [25,26]. BIS measures three areas: disease awareness (BIS-AoI), symptom relabeling (BIS-RoS), and the content that needs treatment (BISNT). BCIS measures self-reflection (BCIS-SR) and selfdeterministic items (BCIS-SC). The combined results of these two questionnaires reflect higher cognitive insights, which means the cognitive insight of patients with schizophrenia is normal. It has not been affected, so cognitive insight is independent.

We now know that TOM has defecated, so there are two specific components under TOM, namely cognitive component and an affective component. Brothers, L, Ring distinguished between "affective" (i.e., It means that it is related to emotions, feelings, and society, to understand the emotions of others) and "cognitive" (i.e., refers to it is related to inference and cognition.) theory of mind [29]. Champagne-Lavau and Stip studied the role of ToM and executive function (EF) in understanding various practical phenomena in patients with schizophrenia, namely transfer, inhibitory control, and cognitive flexibility [27]. The results showed that after excluding the effect of executive function, there are differences in the patients' performance and the control group on actual tasks. But after controlling the role of ToM, the difference exists in the understanding of non-idiomatic metaphors, not in the understanding of indirect speech acts and idiomatic metaphors. This supports that everyone's TOM is different. Still, for patients with schizophrenia, when they understand words or other people's emotions, they will be less able to understand language and non-verbal or extreme language expressions due to impaired "affective theory of mind". Sincere, deceptive, and satirical communicative behavior produces errors. By studying patients with limited anterior peritoneum (VM), prefrontal lobe damage further supports that the theory of mind is different in cognitive and affective TOM [28]. Based on these findings, it can be determined that the behavioral defects of individuals with local VM damage are due to the defect to the affective theory rather than the cognitive defect to the theory of mind. The affective TOM is impaired, but the cognitive TOM is not. Shamay-Tsoory et al. examined the relationship between the empathy scores of schizophrenic patients and healthy people and the performance of cognitive flexibility tasks that assess dorsolateral and orbitofrontal function [30]. The results showed that compared with healthy controls, only affective empathy abilities of patients with schizophrenia were significantly impaired. Patients with schizophrenia exhibit impaired affective ToM using facial processing tasks, including judging and marking facial expressions, recognizing memories of strange faces, and facial recognition. The affective ToM performance of patients with schizophrenia reveals the negative correlation between facial emotion recognition and negative effects [31]. Considering the role emotions play in the process of representing others, the result shows that compared with healthy controls, patients with schizophrenia suffer more from affective TOM rather than cognitive TOM.

Regarding the specific situation where the affective TOM of schizophrenia patients was affected, Taiwan conducted an Eyes test to include schizophrenia patients, and healthy people participated in this study. Studies have found that the affective impairment of affective ToM capabilities may cause the impaired social behavior observed by patients. Also, performance patterns may be related to mental state disorders. The hypothetical connection between mental state and facial recognition is supported by reaction time and accuracy $[31,33]$. Compared with the control group, patients with schizophrenia spent a longer time and were unable to recognize specific mental states from their eyes' emotional expression. Correlation analysis shows that in patients with schizophrenia, there is a significant correlation between the poor recognition performance of facial emotions and the severity of affective symptoms. These results indicate that schizophrenic patients have problems with emotion recognition, and their response errors may be related to social interaction problems. The affective impairment of ToM is the reason for the impaired social behavior observed in patients with schizophrenia. This supports that it is the affective TOM 
that causes schizophrenic patients to be unable to judge their emotions.

\section{UNDER- AND OVER-MENTALIZING IN SCHIZOPHRENIA}

Two general reviews and numerous mentalizing research have demonstrated that most of the published studies report ToM impairment existing in early and chronic schizophrenia [33,34]. Exactly, ToM capacity is performed poorly by individuals with schizophrenia. There is still no enough data about the comprehensive process of ToM impairments on illness. The Results showed that this impairment is at each stage of illness, but there are fewer supports that could determine a deterioration or improvement over time. Green et al. explored the participants' ToM ability stable during the time in prodromal, early (viz., FEP), and chronic phases of schizophrenia. In the study by green et al.., it was found that the performance of chronic schizophrenia patients was greatly poorer than that of early schizophrenia patients. Likewise, the whole performance of patients with chronic schizophrenia was poorer than all ToM subscales (first-order affective, second-order cognitive, and second-order affective) [34].

As it has been evidenced that paranoid schizophrenia patients have a great deal of delusion when their illness is ongoing. Hence, we are about to explore its relationship with the overmentalising and undermentalising of ToM. Montag et al. illustrated the varied responses in individuals with overmentalising and undermentalising. They found the interrelationship in over-and under-mentalising with positive and negative symptoms in schizophrenia, which is the key point that we will mainly discuss in this paper [35]. Different symptoms or the situation of no ToM errors are related to overmentalising and undermentalising. Overmentalizing is defined as a tendency in an individual who excessively gather the intentions or the meaning thought by their own towards to others, and Undermentalizing is defined as the degeneration of mentalising ability in individuals. In other words, although individuals have no problem in mental states, they are difficult to apply their social knowledge, 'reduced mentalizing'. An individual with overmentalising or undermentalising is hard to make a good rapport with people surrounding him. For example, a person who is overmentalising would think too much about others, and sometimes these thoughts are unrealistic or absurd, which may greatly make the feelings of others uncomfortable. Besides, a person with undermentalising could be estranged from people around him. For instance, the undermentalising person does not exactly consider others' real intentions and then make a thing worse. This experiment aimed to explore the relationship between clinical symptoms and specific
ToM error types in early and chronic schizophrenia [34].

Firth indicated that patients who have strikingly negative or disorganized symptoms and developmental onset of the disease might be short of functional concept of mental states such as beliefs or intentions ('undermentalizing'). In contrast, patients who have paranoid symptoms would 'overmentalize', e.g., unduly converge insignificant intentions or self-identified meaning to others and infer behavior on the fundamental wrong beliefs [36]. In this study, Movie for Assessment of Social Cognition (MASC), an institution to professionally read and study adults' minds, provides a short movie relating to real-social interactions for a large but different validation sample of participants, using plenty of multiple choices. Shown in this movie episode is the interaction of four characters gathering together for some daily activities. Then, participants must attempt to understand the mental states of characters and answer those multiple-choice questions. Most of the questions about the mental states are perplexed and have difficulties in mind reading.

The ToM difficulty of individuals with schizophrenia is associated with their previous experience and abnormal integration. In the study by Valerian Chambon et al., researchers entered into the mentalizing impairments in participants with schizophrenia. The impairments were divided into two aspects to explore the difference respectively: one is the scope (basic versus superordinate); The other one is the goal'(non-social versus social) of intention [37]. In addition, it has also been explained through the abnormal integration of visual information and previous experience. It was found that patients who performed poorly in inferring intentions were due to patients excessively rely on previous expectations and inconsistent visual evidence. This abnormal interaction could predict the severity of positive symptoms. They believed that this improper interaction might signal a disorder or disturbance in the inferential mechanism (enables the integration of sensory evidence into previous beliefs to exactly infer other people's intentions. Such a disturbance could be similar to a paranoid (overmentalising) interpretation of other people's goals [37]. All in all, most of the ToM subprocesses impair the patients with early schizophrenia, while they remained higher in the chronic period of the illness. Subsequently, it is presented that 'overmentalizing' could be observable in severe paranoid stages, while 'undermentalizing' could dominate in remission [38]. Shown at the end are the two abnormal aspects of the interaction between previous expectations and sensory sensation. 


\section{NEURAL DYSFUNCTION OF TOM IN SCHIZOPHRENIA}

The weakness of ToM in patients with schizophrenia can be explained by neural dysfunction. Neural activation is closely related to ToM and social reference. Ochsner, in his paper, integrated preexisting studies and knowledge on social-emotional processing stream and subdivided the process into five constructs, (i) Acquisition of social-affective values and responses referring to learning social stimuli of positive outcomes, with the related neural systems amygdala and striatum; (ii) recognition of and response to social-affective stimulus, functioned by the amygdala, striatum, and cortical regions around the superior temporal sulcus; (iii) Embodied Simulation or Low-Level Mental State Inference, the basic process of integrating the mean of social stimulus; (iv) High-level mental state/trait inference, people begin dealing with more sophisticated mental states, such as the false-belief task, with the support from medial PFC regions; (v) Context-sensitive regulation, or the ability to make judgments or behavior to others in an appropriate way [39]. Patients with schizophrenia have deficits in the third and fourth constructs. On the third construct, people embody their reaction to a stimulus by both cognitive judgment and physical expressions; and for the fourth one, people analysis the information given by stimuli in the third stage and perform more complex perspective and behavior (including ToM processing). Different brain regions support each construct. When people sense others' perspectives, feeling, and behavior, the brain regions such as the mirror in the prefrontal portion and the $\mathrm{mPFC}$ region are activated. But for people who lack the ability of ToM, such as patients with Autism and schizophrenia, abnormal neural activation in these regions is observed, explaining their defect in reacting to different social stimuli and ToM processing.

There's a certain area of the brain that takes charge of ToM processes. According to Novel theory, brain regions represented by the right temporoparietal junction (rTPJ) are obviously active during ToM processing [40]. These regions take long-term and shortterm social information from the memory storage part, such as the hippocampus, to support analysis of other persons' perspectives, emotions, and attitudes. Therefore, integration of memory is also a significant measure in ToM processing. In Bitsch and her colleague's research, they tested patients with schizophrenia and a healthy control about their socialdecision making and their neural activity conditions. Participants are introduced to competitive or cooperative patterns and are supposed to decide whether to cooperate or defect. The difference of defective choices on the different patterns is measured, with a positive score indicating the participant's ability to understand the social environment (i.e., being competitive to competitive patterns while being cooperative to cooperative patterns). The results showed that patients with schizophrenia, who earned lower scores on the decisions, present weaker neural responses in the rTPJ region. The connection between rTPJ and the hippocampus is also a significant factor affecting participants' performance: compared to healthy control, patients have a weaker connection between rTPJ and hippocampus. This result suggests that patients with schizophrenia are not good at integrating their memory, which results in weakness in ToM processing.

Another important aspect of ToM is perspective taking, which refers to thinking about others' perspectives. People tend to spontaneously think of others' perspectives even if it is not meaningful; however, patients with schizophrenia seem to lack this ability - it is suggested by an experiment by Kronbichler and colleagues [9]. The experiment also found, for healthy control, the neural activities in the occipital area, especially the right occipital area, are significantly different when they are or not taking others' perspective; however, the patients with schizophrenia do not show significantly different neural activities in this mental area for different scenarios. In sum, (i)patients with schizophrenia are weaker in socialemotional processing because of dysfunction in the mirror system in the prefrontal portion and mPEC region of mind; (ii)brain region regarding memory management is closely related to ToM processing, so patients' weak connectivity between the hippocampus(in charge of memory) and rTPJ region(in charge of integrating information for ToM processing) causes obstacles in ToM processing; (iii)patients with schizophrenia are less spontaneous at perspective-taking processes, related to neural dysfunction in the occipital area.

\section{LIMITATION AND FUTURE DIRECTION}

From a previous review of existing studies, we found that patients with schizophrenia have a deficit in ToM processing, and this deficit is related to dysfunction of certain brain areas. However, the relationship between the deficit in ToM processing and brain dysfunction dysfunction is not clear yet. When patients process ToM, abnormal neural and brain activity can be observed, but it is unsure that this abnormality prevents patients from comprehending information like healthy controls. And the interrelationship between each dysfunctional brain area that may influence ToM processing in patients is not determined. Existing studies are still on the level of exploring the basic principle of ToM (i.e., how it is works in healthy control, how patients process differently, and the supporting body part) by horizontal comparison between patients and healthy control, and 
not yet reach the solution supporting patients' social interaction. Future studies can be focused on the possibilities of treatment or exercise improving ToM processing. Longitudinal studies are necessary to study the natural changes in neural activity and ToM development in patients and the human intervention's consequence.

\section{CONCLUSION}

To recapitulate, this paper has firstly presented the large difference between cognitive incite and cognitive ToM, showing that the behavioral deficits of persons with local VM damage are not associated with the cognitive (i.e., all the mental activities relating to thinking, knowing, remembering, and communicating) impairment to the ToM, but the impairment to the affective(i.e., it is about one's emotions and feelings) theory. Discussed in the next are the positive symptoms and negative symptoms in individuals with schizophrenia and how they affect patients' social mechanisms (such as the attitude toward others, language function, perception, emotion, and daily behavior). Then, we determine some information about the relation in ToM impairment, which is in individuals with early stage and chronic stage, respectively. Different mentalising in patients (such as 'overmentalising' and 'undermentalising') could also influence the level of ToM. Moreover, persons with paranoid schizophrenia could be greatly varied in "overmentalising' and 'undermentalising'. Some given data also indicated that abnormal integration and exaggerated expectations would also lead to the ToM impairment. Neural disorder relating to the deficit of ToM in individuals with schizophrenia is also interpreted. There is much weakness on ToM in schizophrenia patients, which is related to neural dysfunction. After that, a certain area in the brain, the right temporoparietal junction(rTPJ), is explained through some studies and is found the apparent relation with ToM processing. But due to the insufficient data of interrelationship between the deficit in ToM and brain areas dysfunction, this field should still be further explored (like providing more efficient means that can cure dysfunctional brain areas and enhancing ToM processing). In the future, more studies could concentrate on the therapy of dysfunctional brain regions or the practical means to enhance ToM processing. What's more, subsequent studies need to explore more data about neural activity, ToM development, and human intervention.

\section{AUTHORS' CONTRIBUTIONS}

Authors contributed equally.

\section{REFERENCES}

[1] Flavell, J. \& Miller, P. (1998). Social cognition. In D. Kuhn \& R. Siegler (Eds.), Cognition, perception, and language. (5th edn), Handbook of child psychology, Volume 2 (pp. 851-898). New York: Wiley.

[2] J Dunn, J. (1994). Changing minds and changing relationships. In C. Lewis \& P. Mitchell (Eds.), Origins of an understanding of mind (pp. 297310). Hillsdale, NJ: Lawrence Erlbaum Associates.

[3] Leslie, A. (1994). ToMM, ToBY and Agency: Core architecture and domain specificity. In L. Hirschfeld \& S. Gelman (Eds.), Mapping the mind: Domain specificity in cognition and culture (pp. 119-148). Cambridge: Cambridge University Press.

[4] Gopnik, A. \& Wellman, H. (1994). The theorytheory. In L. Hirschfeld \& S. Gelman (Eds.), Mapping the mind: Domain specificity in cognition and culture (pp. 257-293). New York: Cambridge University Press.

[5] Harris, P. L. (1991). The work of the imagination. In A. Whiten (Ed.), Natural theories of mind: The evolution, development, and simulation of everyday mindreading (pp. 283-304). Oxford: Blackwell.

[6] Russell, J. (1997). How executive disorders can bring about an inadequate 'theory of mind'. In J. Russell (Ed.), Autism as an executive disorder (pp. 256-304). New York: Oxford University Press.

[7] Kronbichler, L., Stelzig-Schöler, R., Pearce, B., Tschernegg, M., Said-Yürekli, S., Crone, J.S., Uscatescu, L.C, Reich, L.A., Weber, S., Aichhorn, W., Perner, J., Kronbichler, M. (2019). Reduced Spontaneous Perspective Taking in Schizophrenia. Psychiatry Research: Neuroimaging, 292(2019), 512.

[8] Snyder, S.H., Banerjee, S.P., Yamamura, H.I., \& Greenberg, D. (1974). Drugs, neurotransmitters, and schizophrenia. Science, 184, 1243-1253.

[9] Weinberger, D.R. (1987). Implications of normal brain development for the pathogenesis of schizophrenia. Archives of general psychiatry, 44, 660-669.

[10] Murray, R.M., O'Callaghan, E., Castle, D.J., \& Lewis, S.W. (1992). A neurodevelopmental approach to the classification of schizophrenia. Schizophrenia bulletin, 18, 319-332 
[11] Bloom, F.E. (1993). Advancing a neurodevelopmental origin for schizophrenia. Archives of general psychiatry, 50, 224-227

[12] Harvey, P.D., White, L., Parrella, M., Putnam, K.M., Kincaid, M.M., Powchik, P, Mohs, R.C., \& Davidson, M. (1995). The longitudinal stability of cognitive impairment in schizophrenia: MiniMental State scores at one- and two-year followups in geriatric inpatients. British journal of psychiatry, 166, 630-633.

[13] Green, M.J., \& Phillips, M.L. (2004). Social threat perception and the evolution of paranoia. Neuroscience biobehavioral reviews, 28, 333-342.

[14] Williams, L.M., Loughland, C.M., Gordon, E., \& Davidson, D. (1999). Visual scanpaths in schizophrenia: Is there a deficit in face recognition? Schizophrenia research, 40, 189-199.

[15] Addington, J., Penn, D., Woods, S.W., Addington, D., \& Perkins D.O. (2008). Facial affect recognition in individuals at clinical high risk for psychosis. British journal of psychiatry, 192, 6768.

[16] Kucharska-Pietura, K., David, A.S., Masiak, M., \& Phillips, M.L. (2005). Perception of facial and vocal affect by people with schizophrenia in early and late stages of illness. British journal of psychiatry, 187, 523-528.

[17] Pinkham, A.E., Penn, D.L., Perkins D.O., Graham, K.A., \& Siegel, M. (2007). Emotion perception and social skill over the course of psychosis: A comparison of individuals "at-risk" for psychosis and individuals with early and chronic schizophrenia spectrum illness. Cognitive neuropsychiatry, 12, 198-212

[18] Bentall, R.P., Corcoran, R., Howard, R., Blackwood, N., \& Kinderman, P. (2001). Persecutory delusions: A review and theoretical integration. Clinical psychology Review, 21, 11431192.

[19] Bentall, R.P., \& Swarbrick, R. (2003). The best laid schemas of paranoid patients: Autonomy, sociotropy, and need for closure. Psychology and psychotherapy-theory research and practice, 76, 163-171.

[20] Randall, F., Corcoran, R., Day, J.C., \& Bentall, R.P. (2003). Attention, theory of mind, and causal attributions in people with persecutory delusions: A preliminary investigation. Cognitive neuropsychiatry, 8, 287-294.

[21] Taylor, J.L., \& Kinderman, P. (2002). An analogue study of attributional complexity, theory of mind deficits and paranoia. British journal of psychiatry, 93, 137-140.

[22] Freeman, D. (2007). Suspicious minds: The psychology of persecutory delusions. Clinical psychology review, 27, 425-457.

[23] Beck, A.T., \& Warman, D.M. (2004). Cognitive insight: Theory and assessment. In Amador, X.F., David, A.S.(Eds.), Insight and psychosis: Awareness of Illness in schizophrenia and related disorders(pp.79-88). 2nd Edition. New York: Oxford University Press.

[24] Greig, T.C., Byrson, G.J., \& Bell, M.D. (2004). Theory of mind performance in schizophrenia: Diagnostic, symptom, and neuropsychological correlates. Journal of nervous and mental disease, $192,12-18$.

[25] Birchwood, M., Smith, J., Drury, V., Healy, J., Macmillan, F., \& Slade, M. (1994). A self-report insight scale for psychosis: Reliability, validity, and sensitivity to change. Acta Psychiatrica Scandinavica, 89, 62-67.

[26] Beck, A.T., Baruch, E., Balter, J.M., Steer, R.A., \& Warman, D.M. (2004). A new instrument for measuring insight: The Beck Cognitive Insight Scale. Schizophrenia research, 68, 319-329.

[27] Brothers, L., \& Ring, B. (1992). A neuroethological framework for the representation of minds. Journal of Cognitive Neuroscience, 4, 107-118.

[28] Champagne-Lavau, M., \& Stip, E. (2010). Pragmatic and executive dysfunction in schizophrenia. Journal of neurolinguist, 23, 285296.

[29] Shamay-Tsoory, S.G., Tomer, R., Berger, B.D., Goldsher, D., \& Aharon-Peretz, J. (2005). Impaired "affective theory of mind" is associated with right ventromedial prefrontal damage. Cognitive and behavioral neurology, 18 (1), 55-67.

[30] Shamay-Tsoory, S.G., Harari, H., Aharon-Peretz, J., \& Levkovitz, Y. (2010). The role of the orbitofrontal cortex in affective theory of mind deficits in criminal offenders with psychopathic tendencies. Cortex, 46(5), 668-677.

[31] Irani, F., Platek, S.M., Panyavin, I.S., Calkins, M.E., Kohler, C., Siegel, S.J., Schachter, M., Gur, R.E., \& Gur, R.C. (2006). Self-face recognition and theory of mind in patients with schizophrenia and first-degree relatives. Schizophr Res, 88(1), 151-160. 
[32] Frith, C.D. (1992). The cognitive neuropsychology of schizophrenia. Hillsdale, NJ: Lawrence Erlbaum Associates.

[33] Harrington, L., Siegert, R., \&amp; McClure, J. (2005). Theory of mind in schizophrenia: A critical review. Cognitive Neuropsychiatry, 10(4), 249286. doi :10.1080/13546800444000056

[34] Bora, E., Yucel, M., \&amp; Pantelis, C. (2009). Cognitive endophenotypes of bipolar DISORDER: A meta-analysis of neuropsychological deficits In EUTHYMIC patients and their first-degree relatives. Journal of Affective Disorders, 113(1-2), 1-20. doi: 10.1016/j.jad.2008.06.009

[35] Green, M. F., Bearden, C. E., Cannon, T. D., Fiske, A. P., Hellemann, G. S., Horan, W. P., . . . Nuechterlein, K. H. (2011). Social cognition in schizophrenia, part 1: Performance across phase of illness. Schizophrenia Bulletin, 38(4), 854-864. doi:10.1093/schbul/sbq171

[36] Pickup, G. J., \&amp; Frith, C. D. (1997). 'Theory of mind' and contextual processing in schizophrenia. Schizophrenia Research, 24(1-2), 121. doi:10.1016/s0920-9964(97)82340-7

[37] Montag, C., Dziobek, I., Richter, I. S., Neuhaus, K., Lehmann, A., Sylla, R., . . Gallinat, J. (2011). Different aspects of theory of mind in paranoid schizophrenia: Evidence from a video-based assessment. Psychiatry Research, 186(2-3), 203209. doi: 10.1016/j.psychres.2010.09.006

[38] Chambon, V., Pacherie, E., Barbalat, G., Jacquet, P., Franck, N., \&amp; Farrer, C. (2011). Mentalizing under influence: ABNORMAL dependence on prior expectations in patients with schizophrenia. Brain, 134(12), 3728-3741. doi:10.1093/brain/awr306

[39] Ochsner, K. N. (2008). The social-emotional processing stream: five core constructs and their translational potential for schizophrenia and beyond. Biological psychiatry, 64(1), 48-61.

[40] Bitsch, F., Berger, P., Nagels, A., Falkenberg, I., \& Straube, B. (2018). Impaired right temporoparietal junction-hippocampus connectivity in schizophrenia and its relevance for generating representations of other minds. Schizophrenia Bulletin, 45(4), 934-945. 